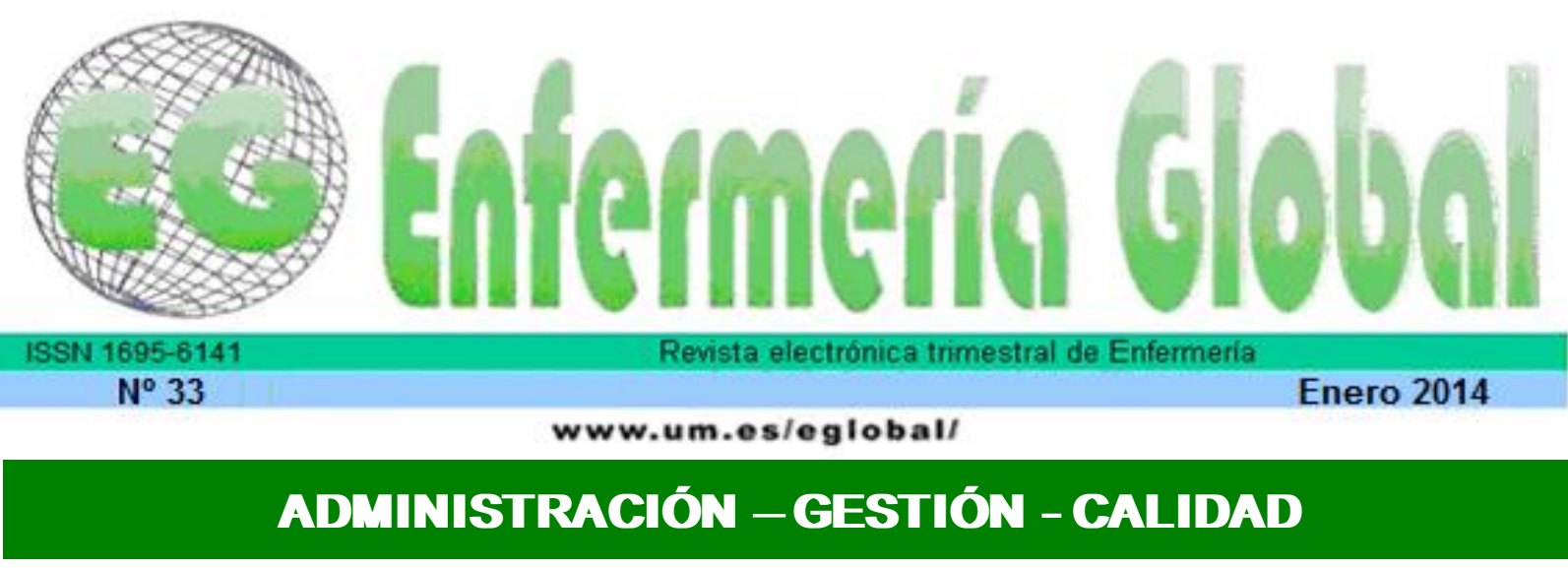

\title{
Accesibilidad a los servicios públicos de salud: la visión de los usuarios de la Estrategia Salud de la Familia
}

Acessibilidade aos serviços públicos de saúde: a visão dos usuários da Estratégia Saúde da Família

Accessibility to public health services: the vision of the users of the Family Health Program in Campina Grande, state of Paraiba, Brazil

\section{${ }^{*}$ Figueroa Pedraza, Dixis **Cavalcanti Costa, Gabriela Maria}

*Doctor en Nutrición. Programa de Post-Graduación en Salud Pública. E-mail: dixisfigueroa@gmail.com **Doctora en Enfermería. Programa de Post-Graduación en Salud Pública.Universidade Estadual da Paraíba. Brasil.

Palabras clave: Acceso a los servicios de salud; Evaluación de los servicios de salud; Atención Primaria de salud

Palavras chave: Acesso aos serviços de saúde; Avaliação de serviços de saúde; Atenção primária à saúde.

Keywords: Access to health services; Health services evaluation; Primary health care.

\section{RESUMEN}

Objetivo: Evaluar, bajo la óptica del usuario, la accesibilidad a la atención primaria de salud de la población adscrita a la Estrategia Salud de la Familia del municipio de Campina Grande, Paraíba, Brasil, estableciendo su relación con la calidad y utilización de los servicios de salud.

Metodología: Estudio transversal en 20 unidades básicas de salud de la familia. La colecta de los datos utilizó cuestionario estructurado con preguntas relacionadas a la percepción de los usuarios sobre la utilización, accesibilidad y calidad de los servicios.

Resultados: De los indicadores de accesibilidad socio-organizacional, el tiempo de espera para marcar una consulta, el tiempo de espera para ser atendido en la realización de la consulta y el tiempo de espera entre marcar la consulta y la atención, fueron relatados como insuficientes por aproximadamente $1 / 3$ de los informantes. Desplazarse a pie hasta las unidades de salud fue destacado. Los indicadores de accesibilidad socio-organizacional presentaron asociación estadísticamente significante con la percepción sobre la calidad del servicio y con la utilización

Conclusión: La evaluación que los usuarios hacen de la calidad de los servicios de salud ofrecidos y la utilización de los mismos está directamente relacionada a los tiempos comprendidos entre la espera para marcar la consulta y la atención. 


\section{RESUMO}

Objetivo: Avaliar, sob a ótica do usuário, a acessibilidade à atenção primária à saúde da população coberta pela Estratégia Saúde da Família no município de Campina Grande, Paraíba, Brasil, estabelecendo sua relação com a qualidade e utilização dos serviços de saúde.

Metodologia: Trata-se de um estudo de corte transversal em 20 unidades básicas de saúde da família. Para a coleta de dados, utilizou-se um questionário estruturado com questões relacionadas à percepção dos usuários sobre a utilização, acessibilidade e qualidade dos serviços prestados.

Resultados: Dos indicadores de acessibilidade sócio-organizacional, o tempo de espera para marcar uma consulta, o tempo de espera para ser atendido na realização da consulta e o tempo de espera entre a marcação da consulta e o atendimento, foram relatados como insuficientes por aproximadamente $1 / 3$ dos informantes. O deslocamento a pé até as unidades de saúde foi destacado. Os indicadores de acessibilidade sócio-organizacional apresentaram associação estatística significante tanto com a percepção sobre a qualidade do serviço quanto com a utilização dos serviços de saúde.

Conclusão: A avaliação que os usuários fazem da qualidade dos serviços de saúde oferecidos e a utilização dos mesmos está diretamente relacionada aos tempos compreendidos entre a espera para marcar uma consulta e o atendimento.

\section{ABSTRACT}

Objective: To evaluate, from the perspective of the user, accessibility to primary health care of the population enrolled in the Family Health Program in Campina Grande, Paraiba, Brazil, establishing its relationship with the quality and use of health services.

Methodology: This is a cross-sectional study in 20 family health units. For data collection it was used a structured questionnaire with questions about user's perception on the use, accessibility and quality of services provided.

Results: The indicators of socio-organizational accessibility, the waiting time for an appointment, the waiting time to be served in the consultation and the delay time between the appointment and the care, were reported as inadequate by approximately one third of informants. The shift to walk to health facilities was highlighted. Indicators of socio-organizational accessibility showed a statistically significant association with both the perception of service quality as with the use of health services.

Conclusion: The quality of health services available and their use is directly related to the times ranging from waiting for an appointment and the care provided

\section{INTRODUCCIÓN}

El acceso a acciones y servicios de salud representa la capacidad del paciente en obtener, cuando necesario, cuidado de salud, de manera conveniente ${ }^{(1)}$. Las reflexiones teóricas apuntan que la utilización de este indicador se inició a partir de la década 60-70 en el escenario norteamericano ${ }^{(2)}$.

A pesar de que el Sistema Único de Saúde (SUS) representa la política de salud de Brasil y haya conseguido a lo largo de su historia avances significativos, encuentra dificultades para garantizar el acceso oportuno y atención de salud de calidad a los brasileños ${ }^{(3)}$. En reconocimiento a esta realidad, el Instituto Brasileiro de Geografia e Estatística (IBGE) divulgó recientemente la obra Um panorama da saúde no Brasil: acesso e utilização dos serviços, condições de saúde e fatores de risco e proteção à saúde ${ }^{(4)}$, con resultados de la situación de salud presentes en la investigación Pesquisa Nacional por Amostra de Domicílios (PNAD) de 2008. La investigación generó informaciones sobre el acceso y utilización de servicios de salud, movilidad 
física y realización de exámenes preventivos de salud. Las informaciones revelaron que el acceso a servicios está aumentando de forma importante, sea en relación a los servicios más simples, como la consulta médica, o en relación a procedimientos más complejos, como la mamografía. Entretanto, a pesar del mayor acceso de la población residente en las regiones más carentes y por los más necesitados, continúan existiendo desigualdades geográficas y sociales.

Con el objetivo de viabilizar la institucionalización de la cultura de evaluación de la atención primaria en el contexto del SUS, fue instituido el Programa Nacional de Melhoria do Acesso e da Qualidade da Atenção Básica (PMAQ). El PMAQ propone un modelo de evaluación de desempeño de los sistemas de salud, en los tres niveles del gobierno, que pretende medir los posibles efectos de la política de salud con miras a subsidiar la toma de decisión, garantizar la transparencia de los procesos de gestión del SUS y dar visibilidad a los resultados alcanzados, además de fortalecer el control social y el enfoque del sistema de salud en los usuarios ${ }^{(3)}$.

Por otro lado, dada la evidencia de la importancia, necesidad, utilidad y pertinencia de la realización y diseminación de los resultados de investigaciones en servicios de salud, surgió el proyecto Desenvolvimento de Metodologia de Avaliação do Desempenho do Sistema de Saúde Brasileiro (http://www.proadess.cict.fiocruz.br). Ese proyecto propone una matriz de dimensiones que deben ser consideradas al definir indicadores, incluyendo la accesibilidad, efectividad, eficiencia y aceptación de los servicios de salud. Destaca que la propuesta de indicadores debe considerar, principalmente, su valor para el monitoramiento del desempeño del SUS ${ }^{(5)}$.

Entendida como componente de la oferta y del proceso de evaluación de servicios de salud $^{(5,6)}$, se considera accesibilidad como el grado de ajuste entre las necesidades de los usuarios y los recursos de la atención de salud. La accesibilidad representa la relación funcional entre los obstáculos en la busca y obtención de cuidados (resistencia) y las capacidades correspondientes de la población para superar tales obstáculos (poder de utilización) ${ }^{(6)}$.

En el concepto de accesibilidad se distinguen dimensiones de orden geográfica (debe medirse en función del tiempo necesario para obtener la asistencia de salud utilizando los medios habituales de transporte), organizacional (refiere los obstáculos que se originan en los modos de organización de los recursos de asistencia a la salud), sociocultural (refiere la apreciación de los fenómenos que determinan la búsqueda de asistencia de salud tales como: percepción sobre el cuerpo y enfermedad, creencias relativas a la salud, tolerancia al dolor y credibilidad en los servicios de salud, entre otros) y económica ${ }^{(7)}$.

La accesibilidad debe ser comprendida, además, considerando los requisitos específicos de análisis que son diferentes para cada nivel de complejidad (atención primaria, media y alta) ${ }^{(8)}$. En relación a la atención primaria, son consagradas, como características importantes, las funciones relacionadas con la continuidad y el vínculo entre los equipos de salud y los usuarios, la integralidad de la atención en sus aspectos de coordinación y cobertura de los cuidados ofrecidos, el enfoque familiar y comunitario y la accesibilidad ${ }^{(9)}$. La accesibilidad representa una dimensión relevante sobre la equidad en los sistemas de salud y uno de los principales atributos de la atención primaria de salud ${ }^{(6,10)}$. 
En el contexto brasileño, el Ministerio de la Salud, dada la necesidad de solucionar los problemas enfrentados por la atención primaria, lanzó en 1994 el Programa Salud de la Familia como forma de re-organización de la atención. Posteriormente, como alternativa para la consolidación de la Estrategia Salud de la Familia, se evidencia la preocupación de Ministerio de la Salud con la evaluación de la atención primaria ${ }^{(11,}$ 12).

Considerando lo expuesto, el objetivo del presente estudio fue evaluar, bajo la óptica del usuario, la accesibilidad a la atención primaria de salud de la población cubierta por la Estrategia Salud de la Familia en el municipio de Campina Grande, Paraíba, Brasil, estableciendo su relación con la calidad y utilización de los servicios de salud.

\section{METODOLOGÍA}

Este estudio es parte de la investigación "Atenção Básica à saúde no município de Campina Grande, Paraíba: avaliação do desempenho e efetividade da Estratégia Saúde da Família e da incorporação das ações de alimentação e nutrição". Este es un estudio de corte transversal con colecta de datos primarios obtenidos entre junio y diciembre de 2010, en el municipio de Campina Grande, Paraíba, Brasil.

El municipio de Campina Grande fue escogido por tener área geográficamente delimitada con unidades básicas de salud de la familia, razonable tradición histórica de organización de servicios de atención primaria de salud, y colaboración entre academia y gestores/equipos de salud. Campina Grande es el segundo mayor municipio del Estado, con población de 376.060 habitantes. Fue municipio pionero en la implantación del Programa Salud de la Familia en 1994 cuando hizo su aparición ${ }^{(13)}$. Posee un sistema de salud compuesto por seis distritos sanitarios con 67 unidades básicas de salud de la familia, con cobertura de $71 \%$ de la población ${ }^{(14)}$.

La unidad de colecta y análisis de los datos fue la unidad básica de salud de la familia, independiente de la cantidad de equipes. Fue considerado el estudio de $30 \%$ de las unidades de salud de la familia del municipio (20 unidades). Fueron seleccionadas unidades considerando los siguientes parámetros: (a) localización en áreas de la ciudad con situación socio-económica semejante; (b) buen padrón relativo al servicio prestado; (c) baja rotatividad de profesionales. Las unidades de salud fueron seleccionadas de forma aleatoria considerando la división por distritos sanitarios con la finalidad de representar los diferentes espacios geográficos/áreas de la ciudad. En cada unidad básica de salud de la familia seleccionada, fue compuesta una muestra intencional de usuarios de todas las atenciones realizadas en un día típico de trabajo.

Para la colecta de datos se utilizó un cuestionario estructurado con informaciones sobre la utilización de los servicios de salud, perfil de los usuarios y su percepción sobre la disponibilidad, accesibilidad y calidad de los servicios prestados. Para la evaluación de la accesibilidad fueron consideradas dos dimensiones, tal como sugerido por Donabedian ${ }^{(15)}$ : a) la accesibilidad socio-organizacional, relacionada con los aspectos de funcionamiento de los servicios que interfieren en la relación entre los usuarios y los servicios; b) la accesibilidad geográfica, relacionada con la distribución espacial de los recursos, la existencia de transporte y la localización de las unidades de salud.

En la accesibilidad socio-organizacional, las variables de estudio consideradas fueron: tiempo de espera para marcar una consulta, tiempo de espera entre marcar la consulta y la atención, tiempo de espera para ser atendido en la realización de la 
consulta y tiempo de duración del atendimiento. Esas variables fueron categorizadas en suficiente, regular, e insuficiente. En la accesibilidad geográfica las variables de estudio consideradas fueron: tiempo necesario para llegar a la unidad de salud $(\leq 15$ minutos; > 15 minutos) y medio utilizado para llegar a la unidad de salud (carro/moto/ómnibus; a pie).

Los indicadores de accesibilidad fueron utilizados como variables explicativas de la utilización de los servicios de salud y de la percepción de los usuarios sobre la calidad de los mismos. La percepción de la calidad consideró las categorías bien/regular o mal. Se consideró utilización de los servicios de salud el recibimiento de atendimiento referido por el usuario en los últimos 30 días anteriores a la entrevista por cualquier motivo relacionado a su salud. Como no utilización se consideró las personas que no procuraron servicios de salud durante el período de estudio, o que procuraron y no recibieron el atendimiento.

El análisis de los datos fue realizado, primeramente, presentando la descripción de distribución de frecuencias relativas de las variables relacionadas a la accesibilidad. En seguida, para evaluar la influencia de estas variables en la utilización de los servicios de salud y en la percepción de la calidad, se estimó la Razón de Chances (RC) y sus Intervalos de Confianza (IC 95\%). Los datos fueron procesados y analizados con la utilización del software Statistical Package for Social Science (SPSS Inc., Chicago, Estados Unidos) versión 15.0. Fueron excluidos de los análisis los registros sin información o cuando el individuo no supo responder.

El proyecto fue evaluado y aprobado por el Comité de Ética en Investigaciones de la Universidade Estadual da Paraíba (parecer nº 0355013300009), respetando las directrices de la Resolución 196/96 del Conselho Nacional de Saúde. Hechos los esclarecimientos, los sujetos firmaron un Termo de Consentimento Livre e Esclarecido, siendo garantizado el sigilo de las informaciones y autorizando la utilización de las informaciones contenidas en el cuestionario para la realización de la investigación.

\section{RESULTADOS}

De un total de 508 individuos de la muestra original, $443(87,2 \%)$ participaron de este estudio. Fueron excluidos 21 registros sin información y 44 referidos a los entrevistados que no supieron responder.

La accesibilidad, que fue evaluada considerando seis indicadores, está representada en la tabla 1. De los indicadores de accesibilidad socio-organizacional, el tiempo de espera para marcar una consulta, el tiempo de espera para ser atendido en la realización de la consulta y el tiempo de espera entre marcar la consulta y la atención, fueron relatados como insuficientes por aproximadamente $1 / 3$ de los informantes. Además, el predominio $(91,6 \%)$ de la forma de desplazarse a pie hasta las unidades básicas de salud de la familia fue destacado. 
Tabla 1: Distribución de frecuencia de los indicadores de accesibilidad a los servicios de atención primaria de salud. Campina Grande, Paraíba, 2010.

\begin{tabular}{|c|c|c|}
\hline Indicadores de accesibilidad & $\mathbf{n}$ & $\%$ \\
\hline \multicolumn{3}{|l|}{ Tiempo de espera para marcar una consulta } \\
\hline Suficiente & 136 & 30,7 \\
\hline Regular & 155 & 34,3 \\
\hline Insuficiente & 152 & 35,0 \\
\hline \multicolumn{3}{|l|}{$\begin{array}{l}\text { Tiempo de espera entre marcar la consulta y la } \\
\text { atención }\end{array}$} \\
\hline Suficiente & 139 & 31,4 \\
\hline Regular & 161 & 36,3 \\
\hline Insuficiente & 143 & 32,3 \\
\hline \multicolumn{3}{|l|}{$\begin{array}{l}\text { Tiempo de espera para ser atendido en la } \\
\text { realización de la consulta }\end{array}$} \\
\hline Suficiente & 127 & 28,7 \\
\hline Regular & 165 & 37,2 \\
\hline Insuficiente & 151 & 34,1 \\
\hline \multicolumn{3}{|l|}{ Tiempo de duración de la atención } \\
\hline Suficiente & 246 & 55,5 \\
\hline Regular & 128 & 28,9 \\
\hline Insuficiente & 69 & 15,6 \\
\hline \multicolumn{3}{|l|}{ Tiempo necesario para llegar a la unidad de salud } \\
\hline$\leq 15$ minutos & 340 & 76,7 \\
\hline$>15$ minutos & 103 & 23,3 \\
\hline \multicolumn{3}{|l|}{ Medio utilizado para llegar a la unidad de salud } \\
\hline Carro/moto/ómnibus & 37 & 8,4 \\
\hline A pie & 406 & 91,6 \\
\hline
\end{tabular}

La calidad de los servicios ofrecidos en las unidades básicas de salud de la familia fue percibida como mal por $10,2 \%(n=45)$ de los entrevistados. Para todos los indicadores de accesibilidad socio-organizacional, la chance (probabilidad) de considerar mal la calidad del servicio fue mayor entre aquellos que clasificaron el indicador como regular y entre aquellos que lo clasificaron como insuficiente. La chance de los usuarios de apuntar la calidad del servicio como mal, al compararse con los usuarios que evaluaron el servicio prestado como bien/regular, fue 33 veces mayor en aquellos que consideraron el tiempo de espera para ser atendido en la realización de la consulta insuficiente ( $R C=32,55$; IC95\% 3,37-5,31); 26 veces mayor en aquellos que consideraron el tiempo de duración de la atención insuficiente $(R C=25,71$; IC95\% 2,31-4,14); 19 veces mayor en aquellos que consideraron el tiempo de espera entre marcar la consulta y el atendimiento insuficiente $(R C=18,96$; IC95\% 2,44-3,96); y 10 veces mayor en aquellos que consideraron el tiempo de espera para marcar una consulta insuficiente $(\mathrm{RC}=10,45 ; \mathrm{IC} 95 \% 2,11-3,18)$. Mayores probabilidades de los usuarios de percibir los servicios ofrecidos como malos, también se encontraron en los casos en que los indicadores anteriores fueron considerados como regular. El tiempo necesario para llegar a la unidad de salud y el medio utilizado no presentaron asociación estadística significante con la percepción de los usuarios sobre la calidad del servicio (tabla 2). 
Tabla 2: Razón de chances (RC) e intervalo de confianza (IC95\%) de los indicadores de accesibilidad a los servicios de atención primaria de salud en relación a la percepción sobre la calidad de los servicios de salud. Campina Grande, Paraíba, 2010

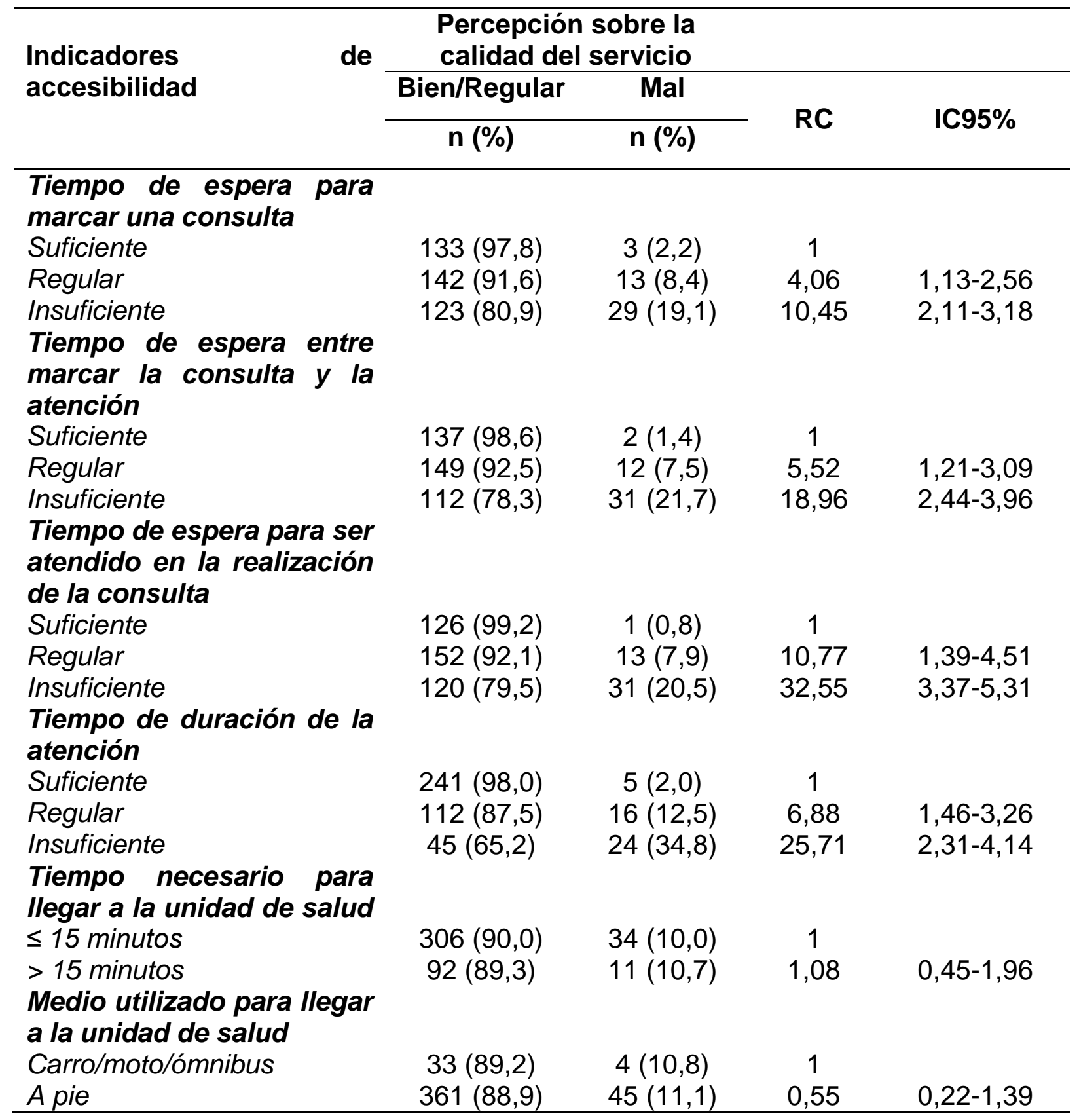

Analizando la utilización de los servicios de salud, se observó que 374 individuos $(84,4 \%)$ utilizaron algún atendimiento en los últimos 30 días. La no utilización de los servicios de salud ocurrió con mayor frecuencia en los usuarios que clasificaron como insuficiente, en relación a los que clasificaron como suficiente, el tiempo de espera para marcar una consulta $(R C=2,33$; IC95\% 1,07-3,39), el tiempo de espera entre marcar la consulta y la atención ( $R C=2,83$; IC95\% 1,58-3,74), el tiempo de espera para ser atendido en la realización de la consulta $(R C=3,25$; IC95\% 1,23-3,38) y el tiempo de duración de la atención ( $R C=2,64 ;$ IC95\% 1,09-2,67). De la misma forma, la no utilización de los servicios de salud presentó mayores chances en los casos de la clasificación regular de los indicadores anteriores referidos a la accesibilidad socioorganizacional (tabla 3). 
Tabla 3: Razón de chances (RC) e intervalo de confianza (IC95\%) de los indicadores de accesibilidad a los servicios de atención primaria de salud en relación a la utilización de los servicios de salud. Campina Grande, Paraíba, 2010.

\begin{tabular}{|c|c|c|c|c|}
\hline \multirow{3}{*}{$\begin{array}{l}\text { Indicadores } \\
\text { accesibilidad }\end{array}$} & \multicolumn{2}{|c|}{$\begin{array}{l}\text { Utilización de los } \\
\text { servicios de salud }\end{array}$} & \multirow{3}{*}{ RC } & \multirow[b]{3}{*}{ IC95\% } \\
\hline & $\mathrm{Si}$ & No & & \\
\hline & n (\%) & n (\%) & & \\
\hline \multicolumn{5}{|l|}{$\begin{array}{l}\text { Tiempo de espera para } \\
\text { marcar una consulta }\end{array}$} \\
\hline Suficiente & $123(90,4)$ & $13(9,6)$ & 1 & \\
\hline Regular & $129(83,2)$ & $26(16,8)$ & 1,91 & $1,02-3,15$ \\
\hline Insuficiente & $122(80,3)$ & $30(19,7)$ & 2,33 & $1,07-3,39$ \\
\hline \multicolumn{5}{|l|}{$\begin{array}{l}\text { Tiempo de espera entre } \\
\text { marcar la consulta y el } \\
\text { atendimiento }\end{array}$} \\
\hline Suficiente & $128(92,1)$ & $11(7,9)$ & 1 & \\
\hline Regular & $131(81,4)$ & $30(18,6)$ & 2,66 & $1,05-3,75$ \\
\hline Insuficiente & $115(80,4)$ & $28(19,6)$ & 2,83 & $1,58-3,74$ \\
\hline \multicolumn{5}{|l|}{$\begin{array}{l}\text { Tiempo de espera para } \\
\text { ser atendido en la } \\
\text { realización de la consulta }\end{array}$} \\
\hline Suficiente & $118(92,9)$ & $9(7,1)$ & 1 & \\
\hline Regular & $135(81,8)$ & $30(18,2)$ & 2,91 & $1,10-1,21$ \\
\hline Insuficiente & $121(80,1)$ & $30(19,9)$ & 3,25 & $1,23-3,38$ \\
\hline \multicolumn{5}{|l|}{$\begin{array}{l}\text { Tiempo de duración del } \\
\text { atendimiento }\end{array}$} \\
\hline Suficiente & $215(87,4)$ & $31(12,6)$ & 1 & \\
\hline Regular & $102(79,7)$ & $26(20,3)$ & 1,77 & $1,01-2,26$ \\
\hline Insuficiente & $50(72,5)$ & $19(27,5)$ & 2,64 & $1,09-2,67$ \\
\hline \multicolumn{5}{|l|}{$\begin{array}{l}\text { Tiempo necesario para } \\
\text { llegar a la unidad de salud }\end{array}$} \\
\hline $\begin{array}{l}\leq 15 \text { minutos } \\
>15 \text { minutos }\end{array}$ & $\begin{array}{l}292(85,9) \\
82(79.6)\end{array}$ & $48(14,1)$ & $\begin{array}{c}1 \\
156\end{array}$ & $088-175$ \\
\hline $\begin{array}{l}\text { Medio utilizado para } \\
\text { llegar a la unidad de salud }\end{array}$ & & & & \\
\hline Carro/moto/ómnibus & $32(86,5)$ & $5(13,5)$ & 1 & \\
\hline A pie & $342(84,2)$ & $64(15,8)$ & 1,19 & $0,45-2,19$ \\
\hline
\end{tabular}

\section{DISCUSIÓN}

La organización de un sistema de salud pasa por el cuestionamiento de las necesidades desde el punto de vista del modelo de asistencia y del usuario ${ }^{(16)}$. El usuario contribuye tanto en la organización del sistema de salud como en la comprensión de las representaciones sociales relacionadas al proceso saludenfermedad y de las dimensiones del acceso al sistema de salud (disponibilidad, accesibilidad, adecuación funcional, capacidad financiera y aceptación) (17). La incorporación del usuario en la evaluación de los servicios de salud ha sido valorada por constituir un indicador sensible de calidad, además de estar potencialmente relacionada a mayor adecuación con el uso del servicio ${ }^{(18)}$. La evaluación que los 
usuarios hacen de los servicios de salud posibilita repensar las prácticas profesionales o modificar la forma de organización, para su perfeccionamiento ${ }^{(19)}$. En otras palabras, la participación de los usuarios en la evaluación de los servicios de salud implica un juicio sobre la característica y calidad de los servicios, lo que facilita información esencial para completar y equilibrar la calidad de la atención ${ }^{(20)}$.

Para los usuarios, los principales obstáculos relacionados a la accesibilidad a las unidades de salud fueron por problemas para marcar consultas, para la realización de consultas y para ser atendido. Las barreras organizacionales como importantes obstáculos de la accesibilidad a los servicios de atención primaria de salud fueron igualmente indicadas por usuarios de unidades de salud en diferentes contextos geográficos: municipio de medio porte del interior de Bahia ${ }^{(21)}$; municipios del Estado de São Paulo con más de 100 mil habitantes ${ }^{(22)}$; tres capitales del Nordeste brasileño, siendo dos metrópolis y un municipio de medio porte ${ }^{(23)}$; cinco municipios de la Región Metropolitana de São Paulo ${ }^{(24)}$. El perfeccionamiento de los manuales de organización del trabajo en las unidades de salud de la familia y la construcción de protocolos enfatizando la agenda y marcación de consultas, la recepción, el cuidado de los usuarios, las rutinas para la atención, la articulación de las acciones, entre otros, puede contribuir positivamente en la accesibilidad a los servicios de atención primaria de salud $^{(21,25,26)}$.

Se destaca el hecho de desplazarse a pie hasta las unidades de salud de la familia, y en 15 minutos o menos, por la mayor parte de los usuarios. El buen desempeño de la accesibilidad geográfica de la Estrategia Salud de la Familia, al colocar las unidades de salud próximas a la vivienda de la población de su área de cobertura, facilita el acceso, la promoción de la equidad y el derecho a la salud ${ }^{(27-29)}$. A pesar de algunos autores indicar beneficio para la descentralización y territorialización en el contexto de la Estrategia Salud de la Familia en la accesibilidad geográfica ${ }^{(21)}$, se destaca la necesidad de reverter el actual diseño de la territorialización para mejorar la organización de la asistencia ${ }^{(27)}$. Se argumenta la apropiación del territorio como proceso dinámico de relaciones, condicionado por el perfil de salud-enfermedad, y no solamente en la lógica normativa de espacios delimitados o en el número de personas para atender las exigencias de funcionamiento ${ }^{(27,30)}$. Los avances en términos de accesibilidad geográfica de la Estrategia Salud de la Familia han sido mostrados en diferentes contextos geográficos $(21,28,31-33)$. Tiempos para desplazarse hasta los servicios de salud, semejantes a los apuntados por los usuarios de Campina Grande, fueron anteriormente reportados en Porto Alegre, Rio Grande do Sul ${ }^{(25)}$ y en Uberaba, Minas Gerais ${ }^{(31)}$.

La percepción que los usuarios tienen sobre los servicios de salud recibidos constituye una de las principales maneras de verificar la satisfacción con los mismos. Altas tasas de satisfacción, como las encontradas en este estudio, es resultado peculiar en la literatura, aconteciendo, incluso, cuando las expectativas sobre los servicios de salud son negativas. Este fenómeno es conocido como efecto de "elevación" de las tasas de satisfacción $(18,34)$. Estos resultados deben ser interpretados con cuidado, pues existen indicios de que los usuarios manifiestan la insatisfacción con los servicios de salud solo cuando consideran los cuidados extremadamente negativos ${ }^{(34)}$. La "elevación" de la tasas de satisfacción ha sido explicada por inconsistencias en el concepto de satisfacción, por falta de sensibilidad de los métodos utilizados para su evaluación y por apropiación de las dimensiones utilizadas. En la evaluación de los servicios públicos de salud, el miedo de los usuarios a perder el derecho a los servicios ofrecidos y la tendencia al agradecimiento 


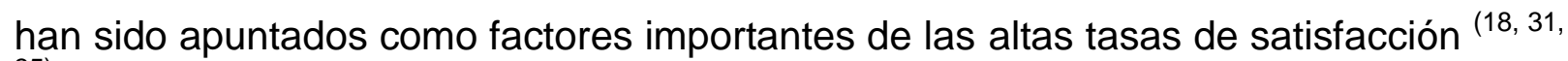
35).

Son dos los grandes grupos de factores relacionados con la satisfacción, uno relativo al servicio y a sus proveedores, y otro inherente a los propios usuarios. La accesibilidad es destacada como una dimensión de los servicios de salud bastante valorizada por los usuarios en la determinación de la satisfacción con los mismos ${ }^{(34,}$ $36,37)$. En revisión sistemática de la literatura, Moreira et al. ${ }^{(38)}$ identificaron 28 aspectos que pueden influir en el acceso y la utilización de los servicios de salud. En ese sentido, predominaron los aspectos relacionados al servicio, agrupados por los autores en la categoría organización y planificación de las acciones de salud, resaltando la importancia de la organización y de la dinámica del proceso de trabajo.

La satisfacción o selección de los servicios de salud marcada por aspectos de la accesibilidad organizacional, más que basada en la accesibilidad geográfica, también ha sido apuntada en otros estudios ${ }^{(31,39-41)}$. Estos resultados revelan la persistencia de problemas en los modos de organización de los recursos de asistencia de salud, perjudicando la longitudinalidad y utilización de los servicios.

La utilización de los sistemas de salud basados en la atención primaria es resultado de la interacción del comportamiento del individuo que busca cuidados, del profesional que lo conduce dentro del sistema de salud y de los recursos disponibles. Entre sus determinantes se destacan las necesidades de salud, las características de los usuarios y las características de los servicios ofrecidos ${ }^{(42)}$. En ese contexto, la relación de los usuarios con el uso de servicios está mediada por la accesibilidad, o sea, la accesibilidad expresa las características de la oferta que intervienen en la relación entre las características de los individuos y el uso de los servicios de salud ${ }^{(6)}$.

De forma semejante a los resultados de este estudio, las barreras de accesibilidad fueron razones atribuidas para la no utilización de ciertos servicios en el barrio de Pau da Lima, Salvador, Bahia ${ }^{(40)}$ y en municipio de Uberaba, Minas Gerais ${ }^{(31)}$. Según Mendoza-Sassi, et al. ${ }^{(43)}$, la distancia entre la vivienda y los servicios de salud tiende a estar asociada con la utilización de los mismos en los casos de países de topografía difícil o de determinados grupos poblacionales particularmente afectados por la distancia. Los autores citan que, contrariamente, esa asociación no acontece en países con una red de atención razonable, a ejemplo de resultados encontrados en Estados Unidos.

\section{CONCLUSIONES}

El concepto de accesibilidad discursa sobre aspiraciones de la población en términos de acciones y servicios de salud, teniendo en los límites de espacio y tiempo bases teóricas de su reflexión. Los indicadores de accesibilidad socio-organizacional, relacionados a los tiempos comprendidos entre la espera para marcar una consulta y el atendimiento, fueron considerados por los usuarios como importantes barreras de acceso de la población a las unidades básicas de salud de la familia. La evaluación que los usuarios hicieron de la calidad de los servicios de salud ofrecidos y la utilización de los mismos estuvo directamente relacionada a los tiempos comprendidos entre la espera para marcar una consulta y el atendimiento. Esos resultados son relevantes para conferir direccionalidad a las intervenciones para mejorar la organización de los servicios de salud en el ámbito del SUS. 
La accesibilidad representa un concepto que va más allá de su contexto geográfico y socio-organizacional. Algunas de esas cuestiones no fueron tratadas en este trabajo. Es de resaltar, por ejemplo, la importancia de la adecuación de los servicios a las necesidades, hábitos y costumbres de la población. Se sabe que el impacto de una intervención está directamente relacionado a su capacidad de atender a los preceptos culturales de la población en que se insertan. En ese contexto, debe garantizarse que el agente comunitario de salud sea habitante de la comunidad, posibilitando actuar como eje mediador entre la comunidad y el servicio de atención primaria de salud. Ese hecho se torna más importante en las condiciones actuales de urbanización y modernización, considerando su influencia en la organización y funcionamiento de la atención de salud y, consecuentemente, en la calidad y utilización de los servicios.

\section{REFERENCIAS BIBLIOGRÁFICAS}

1. Brasil. Ministério da Saúde. Autoavaliação para a melhoria do acesso e da qualidade da Atenção Básica: AMAQ-AB. Brasília: MS; 2012.

2. Instituto Brasileiro de Geografia e Estatística. Pesquisa Nacional por Amostra de Domicílios. Um panorama da saúde no Brasil: acesso e utilização dos serviços, condições de saúde e fatores de risco e proteção à saúde 2008. Rio de Janeiro: IBGE; 2010.

3. Travassos $C$, Martins $M$. Uma revisão sobre os conceitos de acesso e utilização de serviços de saúde. Cad Saude Publ 2004; 20(Supl.2):190-8.

4. Campos CEA. Estratégias de avaliação e melhoria contínua da qualidade no contexto da Atenção Primária à Saúde. Rev Bras Saúde Matern Infant 2005; 5(Supl.1):63-9.

5. Arcêncio RA, Arakawa T, Oliveira MF, Cardozo-Gonzales RI, Scatena LM, Netto $A R$, et al. Barreiras econômicas na acessibilidade ao tratamento da tuberculose em Ribeirão Preto - São Paulo. Rev Esc Enferm USP 2011; 45(5):1121-7.

6. Facchini LA, Piccini RX, Tomasi E, Thumé E, Teixeira VA, Silveira DS, et al. Avaliação de efetividade da Atenção Básica à Saúde em municípios das regiões Sul e Nordeste do Brasil: contribuições metodológicas. Cad Saude Publ 2008; 24(Supl.1): 159-72.

7. Henrique F, Calvo MCM. Avaliação do Programa Saúde da Família nos municípios do Estado de Santa Catarina, Brasil. Cad Saude Publ 2008; 24(4):809-19.

8. Brasil. Ministério da Saúde. Cadastro Nacional de Estabelecimentos de Saúde. Disponível

em:< http://cnes.datasus.gov.br/Mod_Ind_Unidade_Listar.asp?VTipo=02\&VListar $=1 \&$ VEsta do=25\&VMun=250400\&VSubUni>. Acesso em: 23 nov 2011.

9. Kovacs MH, Feliciano KVO, Sarinho SW, Veras AACA. Acessibilidade às ações básicas entre crianças atendidas em serviços de pronto-socorro. J Pediatr 2005; 81(3):251-8.

10. Jesus WLA, Assis MMA. Revisão sistemática sobre o conceito de acesso nos serviços de saúde: contribuições do planejamento. Cien Saude Colet 2010; 15(1):16170.

11. Esperidião MA, Trad LAB. Avaliação de satisfação de usuários. Cien Saude Colet 2005; 10(Supl.):303-12.

12. Uchoa AC, Souza EL, Spinelli AFS, Medeiros RG, Peixoto DCS, da Silva RAR, et al. Avaliação da satisfação do usuário do Programa de saúde da Família na zona rural de dois pequenos municípios do Rio Grande do Norte. Physis 2011; 21(3):1061-76. 
13. Cunha ABO, Vieira-da-Silva LM. Acessibilidade aos serviços de saúde em um município do Estado da Bahia, Brasil, em gestão plena do sistema. Cad Saude Publica 2010; 26(4):725-37.

14. Ibañez N, Rocha JSY, Castro PC, Ribeiro MC, Foster AC, Novaes HDM, Viana ALD. Avaliação do Desempenho da Atenção Básica no Estado de São Paulo. Cien Saude Colet 2006; 11(3):683-703.

15. Souza CFE, Vilar RLA, Rocha NSPD, Uchoa AC, Rocha PM. Acesso e acolhimento na atenção básica: uma análise da percepção dos usuários e profissionais de saúde. Cad Saude Publica 2008; 24(Supl.1):100-10.

16. Pessoto UC, Heimann LS, Boaretto CR, Castro NIE, Kayano J, Ibanhes LC, et al. Desigualdades no acesso e utilização dos serviços de saúde na Região Metropolitana de São Paulo. Cien Saude Colet 2007; 12(2):351-62.

17. Silva Júnior ES, Medina MG, Fonseca ACF, Vilasbôas ALQ. Acessibilidade geográfica à atenção primária à saúde em distrito sanitário do município de Salvador, Bahia. Rev Bras Saude Matern Infant 2010; 10(Supl.):49-60.

18. Medina MG, Hartz ZMA. The role of the family health program in the organization of primary care in municipal health systems. Cad Saude Publica 2009; 25(5):1153-67. 19. Martins GP. Avaliação dos serviços de atenção primária à saúde no município de Uberaba: acessibilidade, utilização e longitudinalidade da atenção [dissertação]. Ribeirão Preto (SP): Universidade de São Paulo; 2006.

20. Escorel S, Giovanella L, Mendonça MHM, Senna MCM. O Programa de Saúde da Família e a construção de um novo modelo para a atenção básica no Brasil. Rev Panam Salud Publica 2007; 21(2):164-76.

21. Espiridião MA, Trad LAB. Avaliação de satisfação de usuários: considerações teórico-conceituais. Cad Saude Publica 2006; 22(6):1267-76.

22. Sofaer S, Firminger K. Patient perception of the quality of health services. Ann Rev Public Health 2005; 26:51-9.

23. Gaioso VP, Mishima SM. User satisfaction from the perspective of acceptability in the family health scenario. Texto Contexto Enferm 2007; 16(4):617-25.

24. Traverso-Yépez M, Morais NA. Reivindicando a subjetividade dos usuários da rede básica de saúde: para uma humanização do atendimento. Cad Saude Publica 2004; 20(1):80-8.

25. Tomasi E, Facchini LA, Thumé E, Piccini RX, Osório A, Silveira DS, et al. Características da utilização de serviços de Atenção Básica à Saúde nas regiões Sul e Nordeste do Brasil: diferenças por modelo de atenção. Cien Saude Colet 2011; 16(11):4395-404.

ISSN 1695-6141

() COPYRIGHT Servicio de Publicaciones - Universidad de Murcia 